The Astrophysical Journal, 195:535-543, 1975 January 15

(C) 1975. The American Astronomical Society. All rights reserved. Printed in U.S.A.

\title{
OBSERVATION OF THE DIFFUSE COMPONENT OF COSMIC SOFT X-RAYS
}

\author{
S. Hayakawa, T. Kato, ${ }^{*}$ Y. Tanaka, and K. Yamashita \\ Department of Physics, Nagoya University, Nagoya, Japan \\ AND \\ J. A. M. Bleeker AND A. J. M. DeERenberG \\ Cosmic Ray Working Group, Kamerlingh Onnes Laboratory, Leiden, The Netherlands \\ Received 1974 April 29; revised 1974 July 22
}

\begin{abstract}
A soft X-ray survey by means of thin-window proportional counters on board a spinning rocket covered a large celestial region with a width of about $60^{\circ}$ in galactic latitude. In the galactic anticenter side the distribution of the soft X-ray intensity is rather smooth and shows a gradual increase with galactic latitude, whereas in the galactic center side the distribution is irregular and shows several enhanced regions. The overall distribution and the energy spectrum are accounted for in terms of a superposition of the following three components: an extragalactic hard component with a power-law spectrum as established at energies higher than $2 \mathrm{keV}$, a galactic soft component which has an exponential spectrum with an apparent temperature of $0.18 \pm 0.02 \mathrm{keV}$, and an extragalactic component with a similar exponential spectrum.

Subject headings: spectra, X-ray - X-rays
\end{abstract}

\section{INTRODUCTION}

Observations of the diffuse component of cosmic soft X-rays have shown a number of characteristic features in contrast to those observed in the energy range above $1.5 \mathrm{keV}$. First, the distribution of the intensity over the celestial sphere is not uniform but patchy. Several enhanced regions have been observed at low galactic latitudes (Bunner et al. 1972, 1973; Davidsen et al. 1972; Palmieri et al. 1972; Yentis, Novick, and Vanden Bout 1972), and there exists a tendency that the intensity gradually increases with latitude in the high galactic latitude region (Bowyer, Field, and Mack 1968; Henry et al. 1968; Bunner et al. 1969; Hayakawa et al. 1971, 1972). Second, the energy spectrum rises more steeply toward low energy than expected from an extrapolation of the power-law spectrum above $1.5 \mathrm{keV}$.

Two apparently conflicting interpretations have been put forward on the origin of the diffuse component of soft X-rays. One extreme is to attribute it to a galactic origin. Gorenstein and Tucker (1972), taking for granted the absence of absorption by the Small Magellanic Cloud (McCammon et al. 1971), assume that the soft X-ray emission region extends to a height of about $800 \mathrm{pc}$ from the galactic plane. The other interpretation is in terms of a superposition of galactic and extragalactic components. This has been discussed extensively by Kato (1972) and by Davidsen et al. (1972). They consider the correlation of the soft Xray intensity with the hydrogen column density as due to the absorption of an extragalactic component and assume the emissivity distribution in the Galaxy to be confined in the gas disk.

A sky survey was conducted with large-area proportional counters on board a free-spinning rocket. Preliminary results of the experiment for the diffuse

\footnotetext{
* Now at Centre d'Études Nucleaires de Saclay, France.
}

component of soft X-rays have been reported elsewhere by one of the authors (Hayakawa 1973a, b). The present paper reports on details of the experiment and results of further analysis.

\section{EXPERIMENT AND DATA ANALYSIS}

The X-ray experiment was launched with a NikeTomahawk rocket from Barking Sands Range, Kauai $(22.05 \mathrm{~N})$, at 0010 local time on 1971 May 26.

The detector and the performance of the experiment were described in detail in a previous publication (Bleeker et al. 1972). The X-ray detector consists of 12 proportional counters, six of which were equipped with $1 \mu$ polypropylene windows and the other six with $5 \mu$ polypropylene windows. The total effective area was $357 \mathrm{~cm}^{2}$ for the $1 \mu$ counters and $361 \mathrm{~cm}^{2}$ for the $5 \mu$ counters. Slat collimators in front of the $1 \mu$ counters define a total geometrical factor of $7.0 \mathrm{~cm}^{2} \mathrm{sr}$ for X-ray energies above 0.5 $\mathrm{keV}$. At lower energies the geometrical factor increases due to X-ray reflection from the collimator blades and becomes $\sim 10 \mathrm{~cm}^{2}$ sr at $280 \mathrm{eV}$ (carbon-K Xrays) and $12.5 \mathrm{~cm}^{2} \mathrm{sr}$ at $180 \mathrm{eV}$ (boron-K X-rays). For the $5 \mu$ counters, stacked-mesh collimators define a geometrical factor of $2.8 \mathrm{~cm}^{2} \mathrm{sr}$ which is energy independent since the collimators are essentially "wall-less." The energy dependence of the geometrical factor for the $1 \mu$ counters has been calibrated at several X-ray energies and is taken into account for the spectral analysis of the diffuse component.

Owing to a relatively large coning angle $\left(21^{\circ}\right)$, a wide galactic latitude band was scanned. The scan path over the celestial sphere slowly changed with time according to the coning motion. The whole observation period was subdivided into seven groups of scan paths. The precession period was rather long, and the field of view perpendicular to the scan path was large 
compared with the change of the scan path within one group; consequently counts obtained for about 20 successive scan paths in each group are added and are regarded as representing the $\mathrm{X}$-ray intensity in that celestial area.

Electrons above several $\mathrm{keV}$ penetrating thinwindow film have occasionally contaminated the soft $\mathrm{X}$-ray measurements, and the electron intensity increases steeply with altitude (Hill et al. 1970; Hayakawa et al. 1973; Kohno 1973; Bunner et al. 1973; Seward et al. 1973). In the present experiment the electrostatic field for the purpose of rejecting such electrons apparently disturbed the ambient plasma and resulted in generation of small pulses in the energy range $0.16-0.37 \mathrm{keV}$. The contribution of an electron component on the presented result can be shown to be negligible on the basis of the following arguments:

i) The observed altitude dependence in the 2-7 keV range. The altitude dependence of the observed counting rate between 122 and $209 \mathrm{~km}$ has been analyzed in five altitude intervals in the energy band $2-7 \mathrm{keV}$. The observed counting rates in each interval are equal within 10 percent, which is consistent with the fact that atmospheric absorption of X-rays is negligible in this range. Observations of electrons in the same altitude range show a severe altitude dependence: Seward et al. (1973) measured a factor 5 intensity difference between 122 and $209 \mathrm{~km}$ altitude for both trapped and parallel components. If this is applied, and considering the statistical weight of our altitude dependence curve, the electron contamination between $2-7 \mathrm{keV}$ cannot be larger than 6 percent at $122 \mathrm{~km}$ and 30 percent at $209 \mathrm{~km}$.

ii) The absolute intensity in the 2-7 $\mathrm{keV}$ range. The measured flux of diffuse X-rays in the interval 2-7 $\mathrm{keV}$ agrees within 30 percent with the well-established flux obtained by many other experimenters in this range (for comparison we took $11 E^{-1.4}$ photons $\left.\mathrm{cm}^{-2} \mathrm{~s}^{-1} \mathrm{sr}^{-1} \mathrm{keV}^{-1}\right)$. This 30 percent excess can be due to electrons, which also cannot be excluded by comparing the $5 \mu$ and $1 \mu$ counter data. Assuming an electron contamination of 30 percent in the energy range $2-7 \mathrm{keV}$, one can compute the electron spectra at lower energy by means of a Monte Carlo method (Hayakawa et al. 1973).

According to these calculations the measured electron spectrum flattens at lower energies, but even when we extrapolate the 30 percent excess in the $2-7 \mathrm{keV}$ interval to lower energies with a $E^{-1}$ law as measured by Seward et al. (1973), the electron intensity will constitute only 10 percent near $0.8 \mathrm{keV}$ and 5 percent (for galactic mid latitudes) near $280 \mathrm{eV}$. Hence it has negligible influence on the measured radiation distributions.

Considering that the counts in the energy range below $0.37 \mathrm{keV}$ could be contaminated with the small pulses generated by the application of the electric field, only data obtained while the electric field was switched off-i.e., one-sixth of the total observation time-are adopted for this energy range.

Our counters were also sensitive to ultraviolet radiation. The bright B-type stars $\alpha \mathrm{Cru}, \beta \mathrm{Cru}$, and $\beta$ Cen exhibited pronounced peaks in the pulse-height region below $0.23 \mathrm{keV}$. Contribution of ultraviolet radiation above this energy is negligible. Observed counting rates for these stars are roughly in agreement with those expected from the laboratorydetermined ultraviolet sensitivity of the counters. However, the correction for the contribution of ultraviolet radiation is subject to a systematic error. We therefore discard data in the energy range below $0.23 \mathrm{keV}$.

\section{INTENSITY DISTRIBUTION OVER THE CELESTIAL SPHERE}

The intensity distributions over the celestial sphere are derived for the energy ranges $0.23-0.37,0.37-0.65$, and $0.65-0.9 \mathrm{keV}$, and are shown in figures $1 a, 1 b$, and $1 c$, respectively. Contributions of discrete sources other than the Cygnus Loop and Sco X-1 are still included in the measured intensity. The intensity in units of counts $\mathrm{cm}^{-2} \mathrm{~s}^{-1} \mathrm{sr}^{-1}$ is represented in several steps. One step corresponds to approximately 2 standard deviations. The counts are lumped in $10^{\circ}$ bins in rocket azimuth for figures $1 a$ and $1 c$ in order to obtain meaningful statistics. For figure $1 b$ a cell size of $5^{\circ} \times 5^{\circ}$ has been adopted. Only data obtained with the $1 \mu$ counters are shown in figure 1 .

\section{a) Regions of Enhanced Radiation}

We shall designate the longitude interval $l=$ $270^{\circ} \rightarrow 90^{\circ}$ the center side and the interval $l=$ $90^{\circ} \rightarrow 270^{\circ}$ the anticenter side.

In the center side, the intensity in the energy range $0.37-0.9 \mathrm{keV}$ is enhanced at low latitudes over a wide longitude range (see figs. $1 b$ and $1 c$ ). This is due partly to the contribution of known discrete sources mostly lying near the galactic plane, but enhancements are observed also in several regions of few known sources. Figure $1 a$ shows that such an enhancement toward the galactic center is not evident in the energy range $0.23-0.37 \mathrm{keV}$. In figure $1 b$ one notices several regions of enhancement. The enhancement near $l=350^{\circ}, b=-5^{\circ}$ is due to a soft X-ray source in the vicinity of a bright B-type star, $\lambda$ Sco (Bleeker et al. 1973). The other regions of enhancement are extended and probably not due to single discrete sources. Such an extended enhancement was also detected by other observers (Bunner et al. 1972; Yentis et al. 1972; Palmieri et al. 1972; Davidsen et al. 1972).

Regions of significant enhancement noticed from figure $1 b$ with intensities higher by 2 standard deviations than the surrounding regions are:

$$
\begin{aligned}
& \text { A) } l=20^{\circ}-30^{\circ}, \quad b=10^{\circ}-25^{\circ} ; \\
& \text { B) } l=320^{\circ}-335^{\circ}, b=10^{\circ}-20^{\circ} ; \\
& \text { C) } l=310^{\circ}-315^{\circ}, b=20^{\circ}-40^{\circ} ; \\
& \text { D) } l=355^{\circ}-10^{\circ}, \quad b=-10^{\circ}--20^{\circ} ; \\
& \text { E) } l=25^{\circ}-35^{\circ}, \quad b=-15^{\circ}--25^{\circ} .
\end{aligned}
$$


Region A coincides with the enhanced region near the foot of the North Polar Spur reported by Bunner et al. (1972). The observed excess is significant in the energy range $0.37-0.65 \mathrm{keV}$, whereas it is not in the $0.23-0.37 \mathrm{keV}$ range.

Region B includes the Lupus Loop. The Lupus Loop (supernova remnant $l=330^{\circ}, b=15^{\circ}$ ) has a diameter of 4.5 in radio. The region of the soft X-ray enhancement is much broader than the radio loop. A similar broad enhancement in this region has been reported by Palmieri et al. (1972).

Region $\mathrm{C}$ has a softer spectrum than other enhanced regions and hence possibly located at a distance smaller than $200 \mathrm{pc}$. The enhancement in region $\mathrm{D}$ is not caused by the galactic center sources, because the energy spectrum of this region is markedly softer than that of the center sources. In fact, the intensity of region $\mathrm{D}$ in the energy range $0.9-6.5 \mathrm{keV}$ is smaller by a factor of 5 than that near the galactic plane.

Contribution of discrete sources near the galactic plane to region $\mathrm{E}$ is also negligible, since the intensity increases with galactic latitude.

For the lowest energy range $0.23-0.37 \mathrm{keV}$ the intensity distribution is not available with comparable angular resolution because of poor statistics, but local enhancements do not seem to be pronounced in this energy range. This indicates the distance of most enhanced regions to be several hundreds of parsecs. Accordingly their linear size may be as large as $100 \mathrm{pc}$ on account of their angular sizes of about $10^{\circ}$. Such a large size may be hardly explained as due to a single extended source. Objects such as the North Polar Spur and the Lupus Loop may account for enhancements $\mathrm{A}$ and $\mathrm{B}$. The other enhancements do not seem to be correlated with known radio spurs and supernova remnants (Kato 1972).

\section{b) Latitude Dependence and Correlation with Interstellar Matter}

The X-ray intensity distribution in the anticenter side is much less complex than that in the center side. The intensity increases toward high galactic latitude. This feature is more pronounced as the X-ray energy becomes smaller.

The soft X-ray intensities in the anticenter direction are compared with the hydrogen column density $N_{\mathrm{H}}$ derived from $21-\mathrm{cm}$ radio observations. At low latitudes where $N_{\mathrm{H}}$ varies steeply with latitude, the value of $N_{\mathrm{H}}$ is averaged over the field of view according to the collimator response function. The averaged hydrogen column density is denoted by $\left\langle N_{\mathrm{H}}\right\rangle$. For the correlation study, the X-ray intensities in different regions having the same value of $\left\langle N_{\mathrm{H}}\right\rangle$ are averaged, since the dispersion of these intensities is found to be within the statistical fluctuations. In figures $2 a$ and $2 b$, $\left\langle N_{\mathrm{H}}\right\rangle$ dependences are shown for four energy ranges of the $1 \mu$ counters and for $0.23-0.37 \mathrm{keV}$ of the $5 \mu$ counters. In the range $0.23-0.65 \mathrm{keV}$, a tendency of decreasing X-ray intensity with increasing $\left\langle N_{\mathrm{H}}\right\rangle$ is observed for the range of $\left\langle N_{\mathrm{H}}\right\rangle,(1-10) \times 10^{20}$ atoms $\mathrm{cm}^{-2}$. This is consistent with the interstellar absorption of the $\mathrm{X}$-rays originating outside the absorption layer of the Galaxy, whereas the plateau at larger $\left\langle N_{\mathrm{H}}\right\rangle$ values should be attributed to galactic emission.

\section{ORIGIN OF THE DIFFUSE COMPONENT OF SOFT X-RAYS}

As mentioned in $\S \mathrm{I}$, two different models have been proposed on the origin of the diffuse component of soft X-rays. Since most of our scan paths are nearly parallel to the galactic equator and the latitude range covered is not too wide, the present result is not particularly suited to distinguish between these two models. We shall consider here a two-component model, i.e., a superposition of an extragalactic component which is isotropic and a galactic component associated with the gas disk of the Galaxy.

The correlation function of the soft X-ray intensity with the hydrogen column density is interpreted in terms of two components; an isotropic extragalactic component $I_{e}$, and a galactic component $I_{g}$. If the volume emissivity in the Galaxy is assumed to be proportional to the interstellar hydrogen density, the relation $I_{g}$ vs $N_{\mathrm{H}}$ is expressed as

$$
I_{g}(E)=[q(E) / \sigma(E)]\left[1-\exp \left(-\sigma(E) N_{\mathrm{H}}\right)\right]
$$

where $4 \pi q(E)$ and $\sigma$ are the emissivity and the absorption cross section per hydrogen atom, the latter being adopted from Brown and Gould (1970). Hence, the superposition of the extragalactic and galactic components gives the $N_{\mathrm{H}}$ dependence,

$$
I\left(N_{\mathrm{H}}\right)=I_{e} \exp \left(-\sigma N_{\mathrm{H}}\right)+(q / \sigma)\left[1-\exp \left(-\sigma N_{\mathrm{H}}\right)\right] .
$$

We further assume that the extragalactic component $I_{e}$ consists of a soft component $\left(I_{e s}\right)$ and a hard component $\left(I_{e h}\right)$. The hard component is taken to be the extrapolation of the diffuse X-ray spectrum established in the energy range $2-10 \mathrm{keV}$ which can be expressed as (Bunner et al. 1969)

$$
I_{e h}=11 E^{-1.4} \text { photons } \mathrm{cm}^{-2} \mathrm{~s}^{-1} \mathrm{sr}^{-1} \mathrm{keV}^{-1} \text {. }
$$

Since the contribution of the extragalactic component is negligible near the galactic plane at energies below $0.65 \mathrm{keV}$, the volume emissivity per hydrogen atom of the galactic component $4 \pi q(E)$ can be estimated from the measured energy spectrum. Using equation (1) and assuming an exponential type emission spectrum,

$$
\begin{aligned}
4 \pi q(E)=4.5 & \times 10^{-18} E^{-1} \exp (-E / k T) \\
& \text { (hydrogen atom) })^{-1} \mathrm{~s}^{-1} \mathrm{keV}^{-1}
\end{aligned}
$$

with $k T=0.18 \pm 0.02 \mathrm{keV}$.

Figure $3 a$ presents the fit of the spectrum measured with the $1 \mu$ counters for the low-latitude region, $b=$ $0^{\circ}-30^{\circ}$, in the anticenter side. The measured flux values given for the $1 \mu$ counters are not corrected for the energy-dependent geometrical factor, since an 


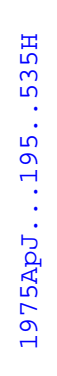

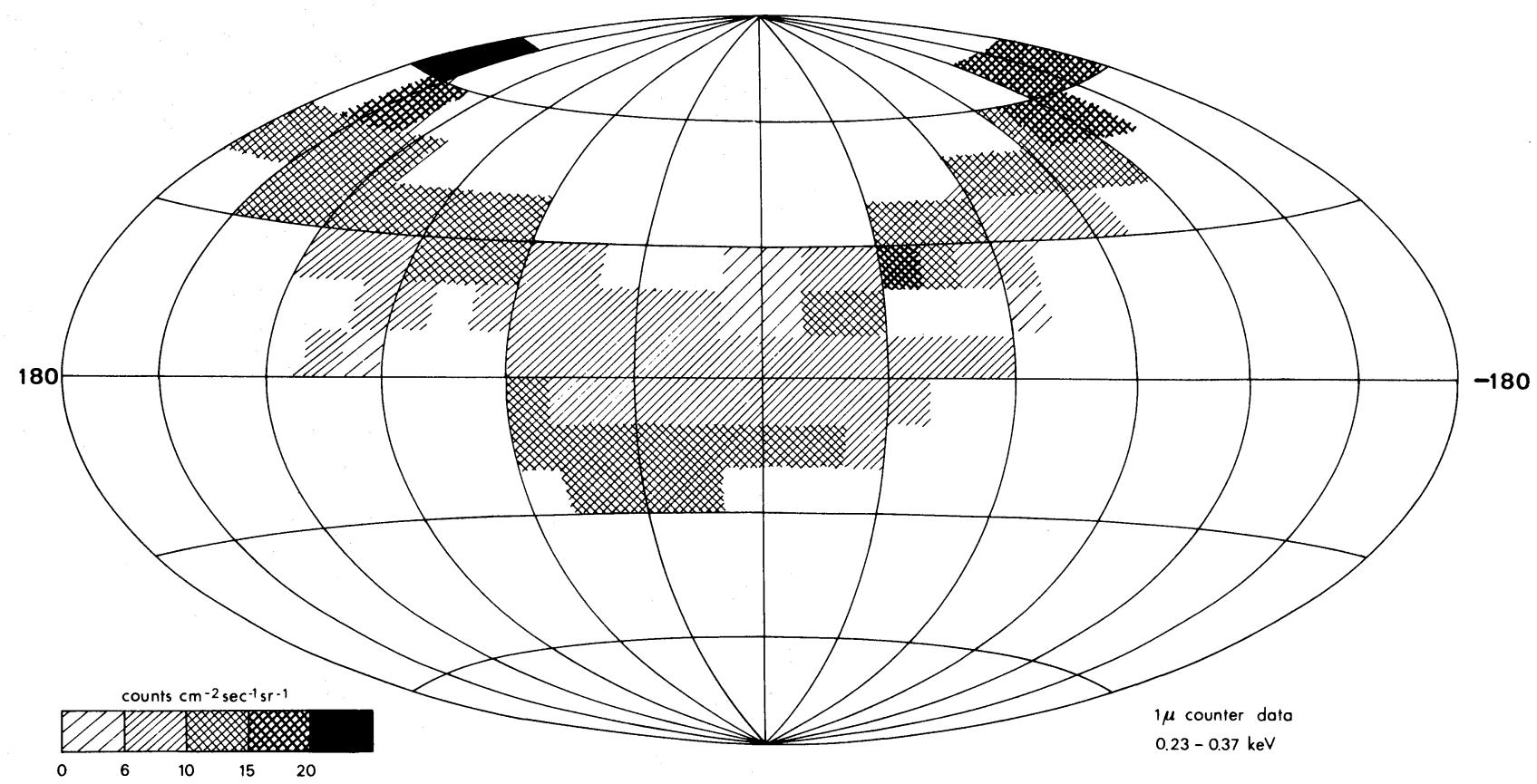

FIG. $1 a$

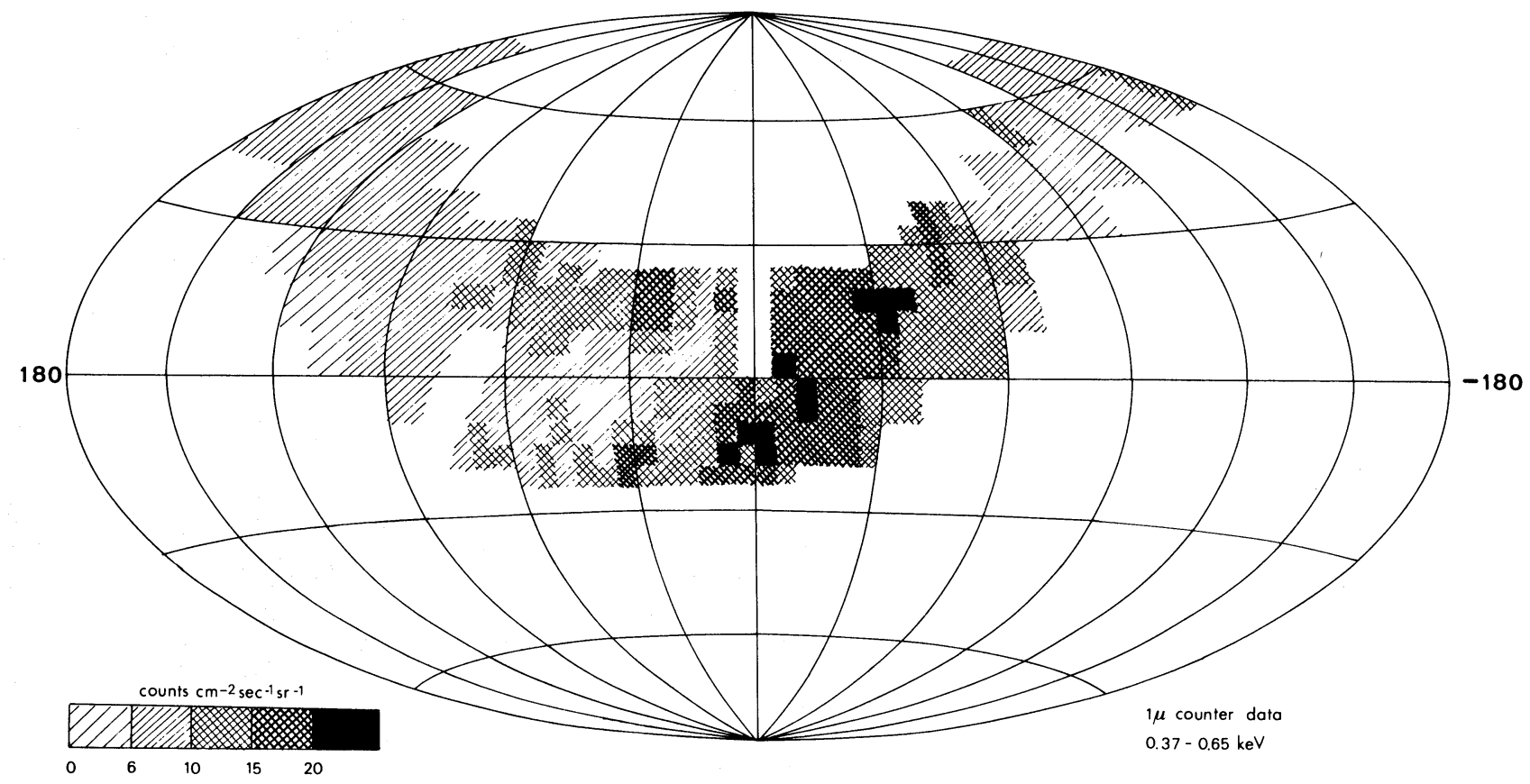

Fig. $1 b$ 


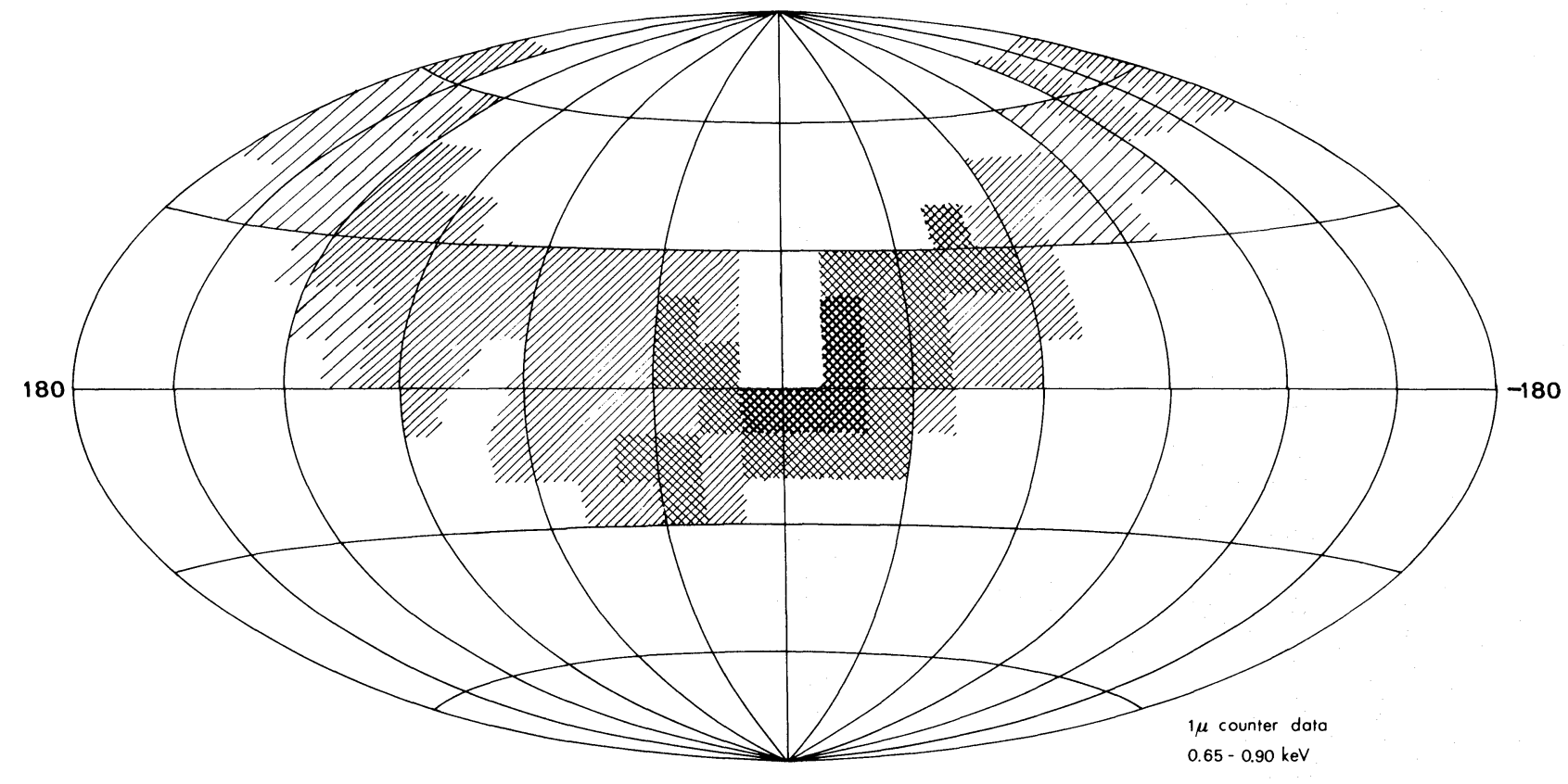

Fig. $1 c$

FIG. 1.-Celestial distributions of the X-ray intensity in three energy ranges, (a) $0.23-0.37 \mathrm{keV}\left(10^{\circ}\right.$ bin), (b) $0.37-0.65 \mathrm{keV}$ $\left(5^{\circ} \mathrm{bin}\right)$, and $(c) 0.65-0.9 \mathrm{keV}\left(10^{\circ}\right.$ bin $)$. The intensity in units of counts $\mathrm{cm}^{-2} \mathrm{~s}^{-1} \mathrm{sr}^{-1}$ is represented in several steps, one step roughly corresponding to two standard deviations.

accurate correction requires the incident spectrum to be known. Instead, the energy dependence has been incorporated in the computation of the model spectra to make the comparison with the observed results appropriate. The averaged hydrogen column density is taken to be $\left\langle N_{\mathrm{H}}\right\rangle=30 \times 10^{20} \mathrm{~cm}^{-2}$. Values of $k T$ outside $0.18 \pm 0.02$ poorly fit the observed spectrum.

The extragalactic component is obtained by subtracting the galactic component given in equation (1) from the observed spectrum in the high-latitude region, $b=50^{\circ}-65^{\circ}$. The result requires an excess soft component $I_{e s}$ in addition to the extrapolation of the power-law spectrum, $I_{e h}$, given by equation (3). $I_{e s}$ can be fitted reasonably by an exponential spectrum,

$I_{e s}=5 \times 10^{2} E^{-1} \exp (-E / k T) \mathrm{cm}^{-2} \mathrm{~s}^{-1} \mathrm{sr}^{-1} \mathrm{keV}^{-1}$

with a $k T$ value of $0.18 \mathrm{keV}$. The resultant spectrum, $I=I_{g}+I_{e s}+I_{e h}$, reproduces the observed one in the high-latitude region with $\left\langle N_{\mathrm{H}}\right\rangle=3 \times 10^{20} \mathrm{~cm}^{-2}$ as shown in figure $3 b$.

Substituting the above derived energy spectra in equation (2), we obtain the intensity for a given value of $\left\langle N_{\mathrm{H}}\right\rangle$. The dependence on $\left\langle N_{\mathrm{H}}\right\rangle$ thus calculated is shown in figure 2. The contributions of respective components are given separately. The summations of these components are in general agreement with the observed result.
Figures $4 a$ and $4 b$ also show the energy spectra measured with the $5 \mu$ counters for the same regions. The dashed curves represent the calculated ones for the same input spectra as derived above. The agreement between the measured and calculated spectra are consistent for both types of counters. This demonstrates that the assumed input spectra are appropriate.

The differential emissivity adopted in equation (4) gives the energy generation rate of the galactic X-rays with energies greater than $E_{0}$,

$$
\begin{aligned}
Q\left(E_{0}\right)= & 4 \pi \int_{E_{0}}^{\infty} E q(E) d E \\
& =1.3 \times 10^{-27} \exp \left(-E_{0} / 0.18 \mathrm{keV}\right) \\
& \left.\quad \operatorname{ergs~s}^{-1} \text { (hydrogen atom }\right)^{-1} .
\end{aligned}
$$

If most of the galactic X-rays are absorbed by interstellar matter, then several percent of the ionization rate required for the heating of $\mathrm{H}$ I clouds can be accounted for (Hayakawa 1973a, $b$ ).

The apparent absence of the absorption by the Small Magellanic Cloud (McCammon et al. 1971) could be accounted for in terms of the soft X-ray emission therefrom, if the emissivity per hydrogen atom is about 3 times higher than that given in equation (4). Even if such high emissivity is assumed for galaxies, a superposition of soft X-rays from individual galaxies still falls short of the intensity of the extragalactic soft X-rays as given in equation (5). 

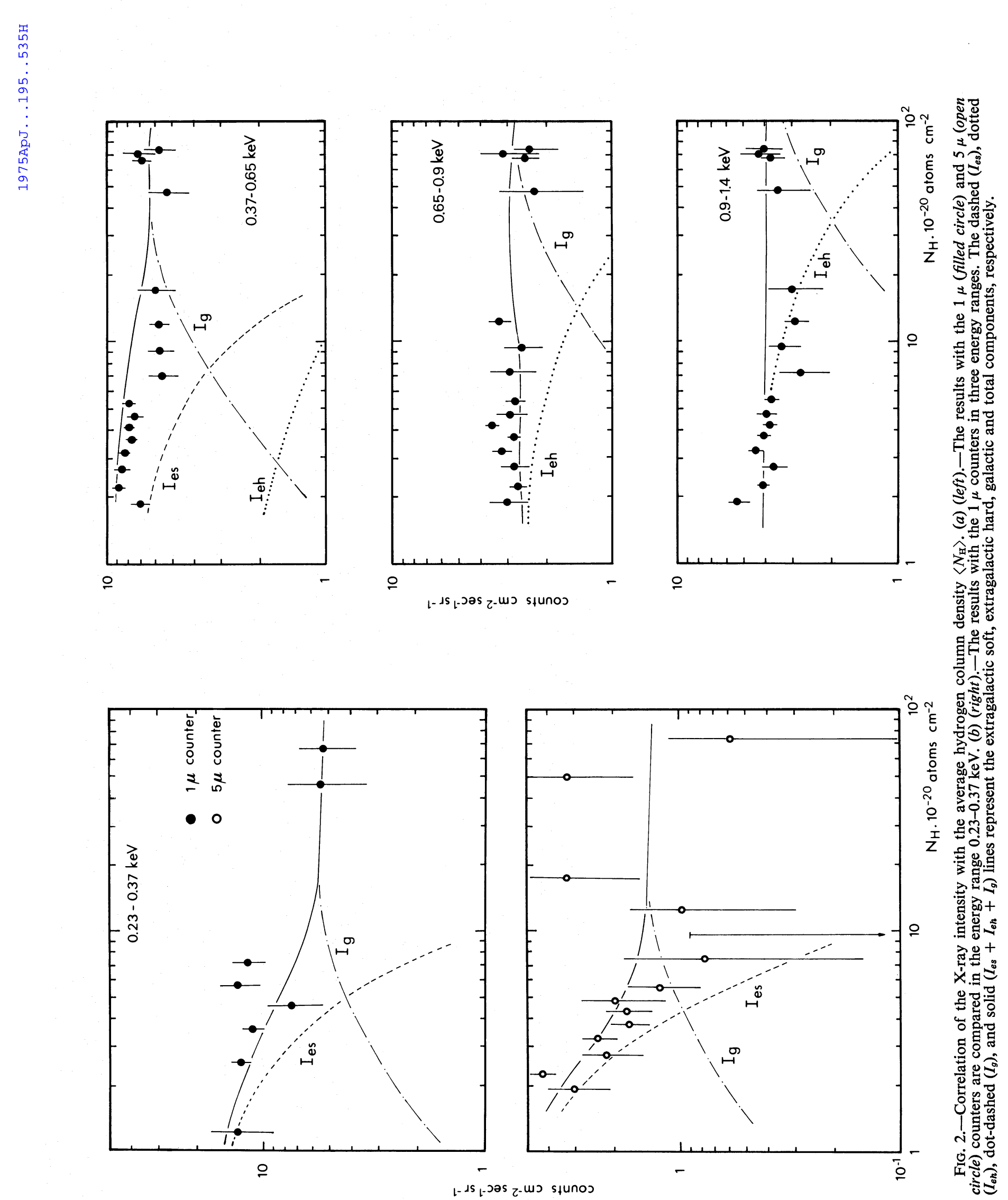

540 


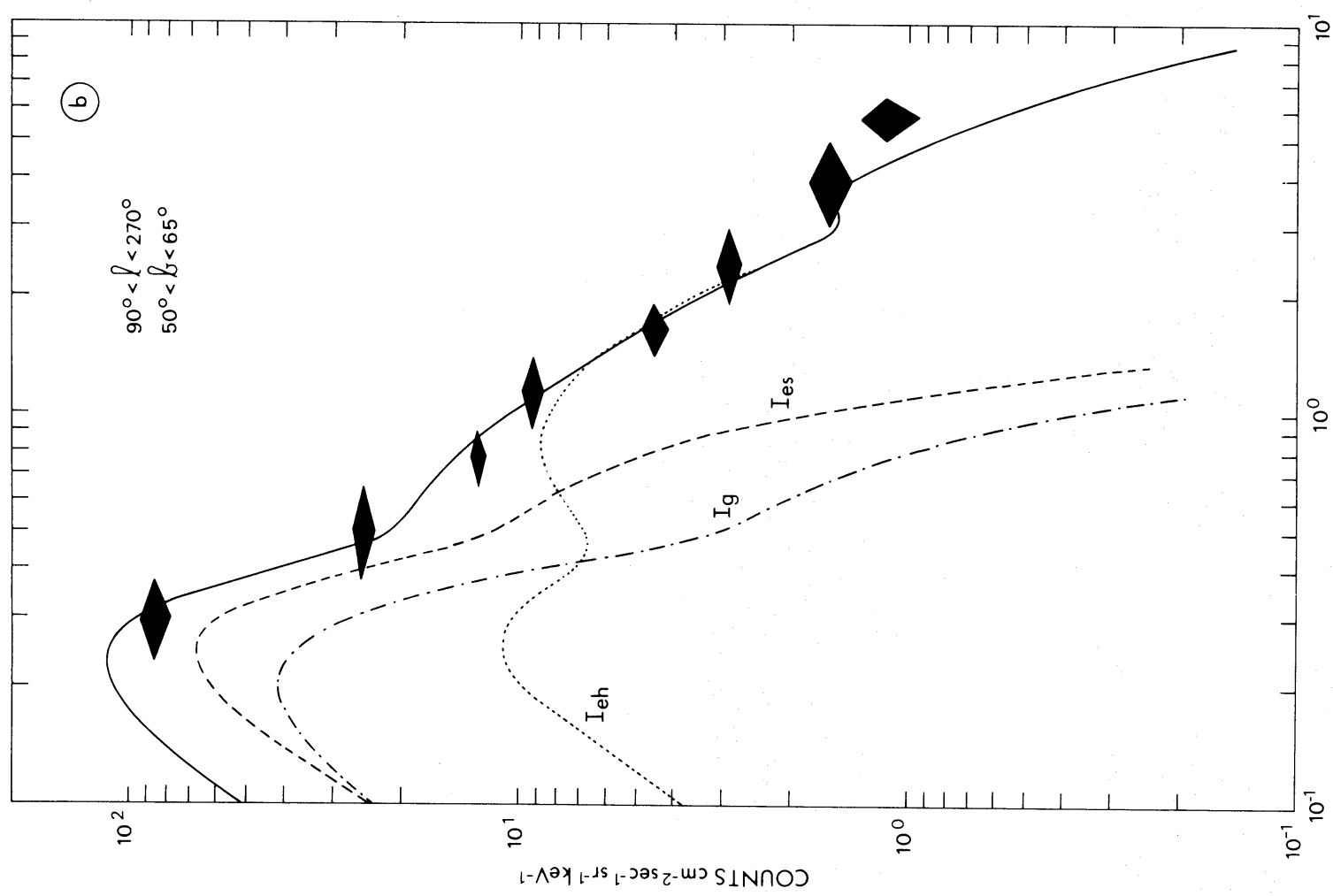

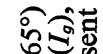

VI

VI

实客

맬요

s.

勇

สั

>

V-

VI: 형

일

을

()

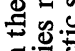

‥

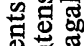

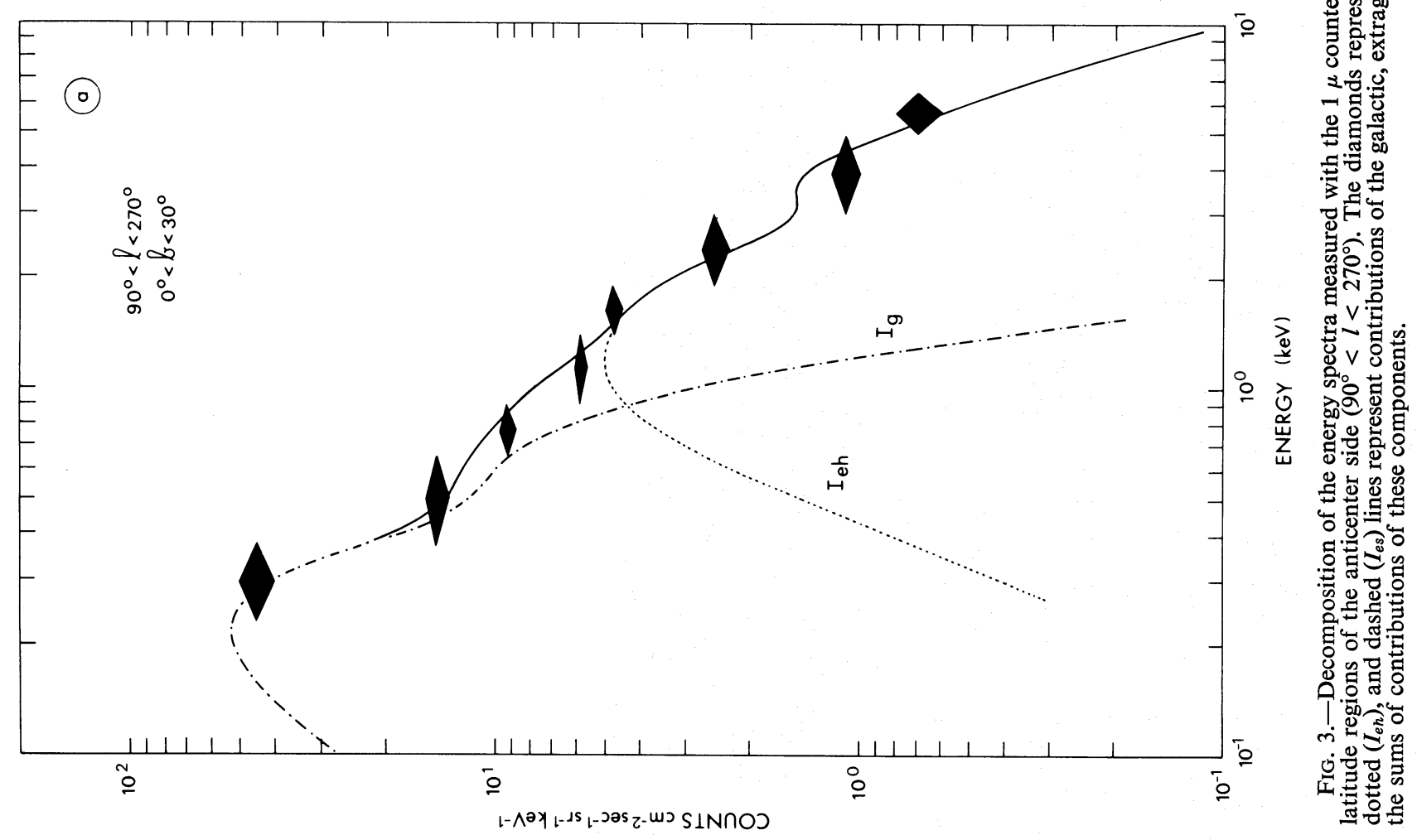



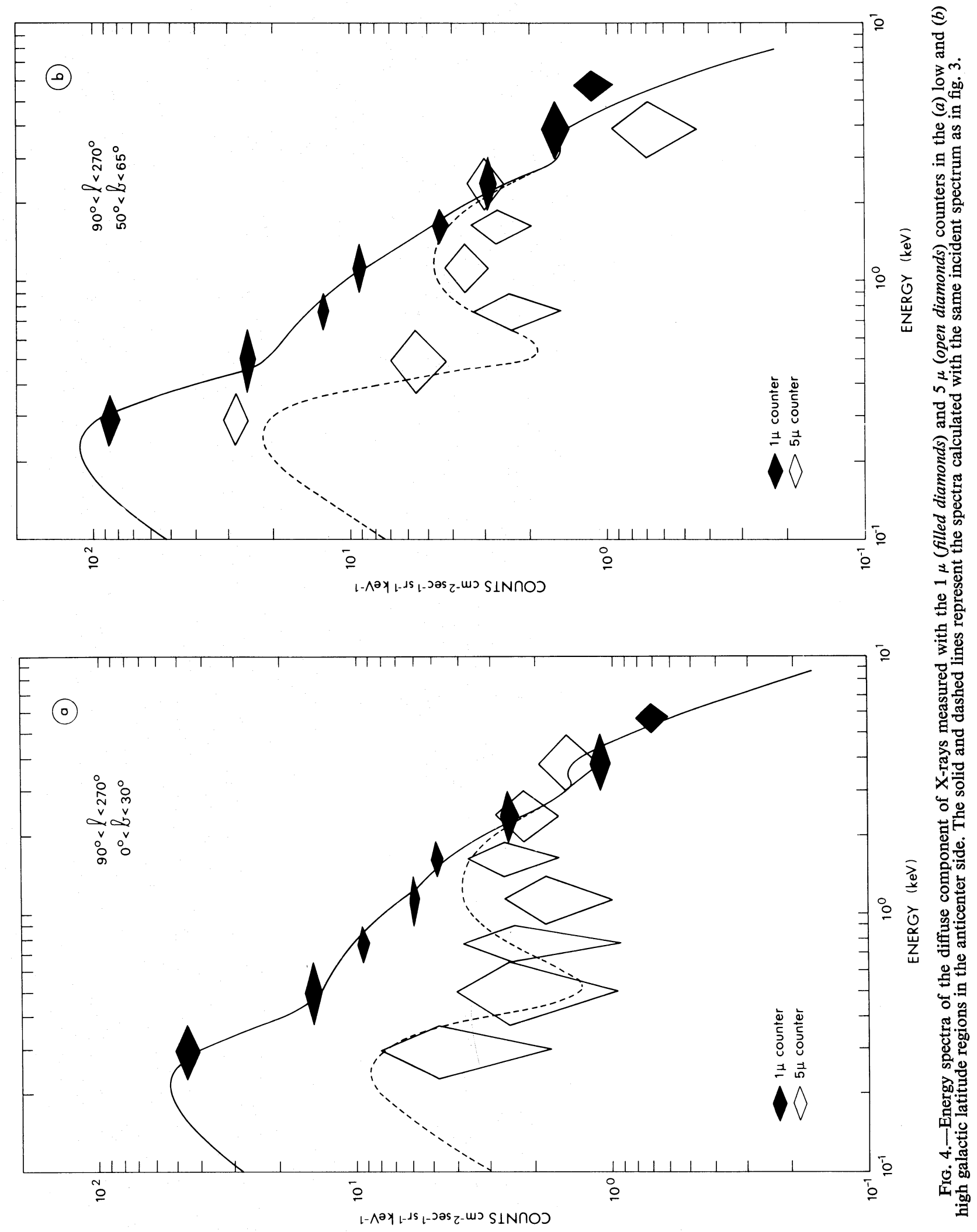
The authors acknowledge discussions with P. A. J. de Korte on the data analysis and express their thanks to Professor H. C. van de Hulst for his efforts in arranging the present experiment and to Professor
D. Mori for his advice on the mechanical design of the payload. They also thank the Royal Dutch Academy of Sciences and Toray Science Foundation for financial support.

\section{REFERENCES}

Bleeker, J. A. M., Deerenberg, A. J. M., Yamashita, K., Hayakawa, S., and Tanaka, Y. 1972, Ap. J., 178, 377.

Bleeker, J. A. M., Deerenberg, A. J. M., Heise, J., Yamashita, K., and Tanaka, Y. 1973, Nature Phys. Sci., 241, 55.

Bowyer, C. S., Field, G. B., and Mack, J. 1968, Nature, 217 32.

Brown, R. L., and Gould, R. J. 1970, Phys. Rev., D1, 2252.

Bunner, A. N., Coleman, P. L., Kraushaar, W. L., and McCammon, D. 1972, Ap.J. (Letters), 172, L67.

Bunner, A. N., Coleman, P. L., Kraushaar, W. L., McCammon, D., Palmieri, T. M., Shilepsky, A., and Ulmer, T. M. 1969, Nature, 223, 1222.

Bunner, A. N., Coleman, P. L., Kraushaar, W. L., McCammon, D., and Williamson, F. O. 1973, Ap. J., 179, 781.

Davidsen, A., Shulman, S., Fritz, G., Meekins, J. F., Henry, R. C., and Friedman, H. 1972, Ap., J., 177, 629.

Gorenstein, P., and Tucker, W. H. 1972, Ap. J., 176, 333.

Hayakawa, S. $1973 a$, Proc. IAU Symposium 55 on $X$ - and Gamma-Ray Astronomy, ed. H. Bradt and R. Giacconi (Dordrecht: Reidel), p. 235.

1973b, Proc. IAU Symposium 52 on Interstellar Dust, ed. J. M. Greenberg and H. C. van de Hulst (Dordrecht: Reidel).
Hayakawa, S., Kato, T., Kohno, T., Murakami, T., Nagase, F., Nishimura, K., and Tanaka, Y. 1973, J. Geomag. Geoelectr., 25, 113.

Hayakawa, S., Kato, T., Kohno, T., Nishimura, K., Tanaka, Y., and Yamashita, K. 1972, Ap. and Space Sci., 17, 30.

Hayakawa, S., Kato, T., Makino, F., Ogawa, H., Tanaka, Y., Yamashita, K., Matsuoka, M., Miyamoto, S., Oda, M., and Ogawara, Y. 1971, Ap. and Space Sci., 12, 789.

Henry, R. C., Fritz, G., Meekins, J. F., Friedman, H., and Byram, E. T. 1968, Ap.J. (Letters), 153, L11.

Hill, R. W., Grader, R. J., Seward, F. D., and Stoering, J. P. 1970, J. Geophys. Res., 75, 7267.

Kato, T. 1972, Ap. and Space Sci., 16, 478.

Kohno, T. 1973, J. Geomag. Geoelectr., 25, 131.

McCammon, D., Bunner, A. N., Coleman, P. L., and Kraushaar, W. L. 1971, Ap.J. (Letters), 168, L33.

Palmieri, T. M., Burginyon, G. A., Hill, R. W., Scudder, J. K., and Seward, F. D. 1972, Ap. J., 177, 387.

Seward, F. D., Grader, R. J., Toor, A., Burginyon, G. A., and Hill, R. W. 1973, preprint.

Yentis, D. J., Novick, R., and Vanden Bout, P. 1972, Ap. J., 177, 365 .

S. Hayakawa, T. Kato, and Y. Tanaka: Department of Physics, Nagoya University, Chikusa-Ku, Nagoya, Japan

J. A. M. Bleeker, A. J. M. Deerenberg, and K. Yamashita: Cosmic Ray Working Group, Kamerlingh Onnes Laboratorium, Nieuwsteeg 18, Leiden, The Netherlands 
